\title{
MÉTHODE DES MICROHABITATS : PRINCIPES ET PROTOCOLES D'APPLICATION.
}

\author{
M. POUILLY (1), Sylvie VALENTIN (1), H. CAPRA (1), V. GINOT (2), Y. SOUCHON (1)
}

(1) CEMAGREF, Laboratoire d'Hydroécologie Quantitative, Division Biologie des Ecosystèmes Aquatiques, 3 bis Quai Chauveau, CP 220, 69336 Lyon Cedex 09, France.

(2) Adresse actuelle : INRA, 75 avenue de Corzent, BP 511, 74203 Thonon-les-Bains, France.

Reçu le 11 octobre 1994

Accepté le 8 juin 1995

Received 11 October, 1994

Accepted 8 June, 1995

\section{RÉSUMÉ}

Cette note expose, à la demande d'utilisateurs potentiels, le protocole précis de mise en oeuvre de la méthode des microhabitats développée au CEMAGREF de Lyon depuis 1985 et adaptée de l'IFIM de l'US Fish and Wildlife Service. Les principes des modèles inclus dans la méthode sont présentés. Les protocoles de mesures sont expliqués en fonction du modèle hydraulique et de la méthode de couplage des informations hydrauliques et biologiques. Les limites d'application sont ensuite exposées.

Mots-clés : méthode des microhabitats, habitat des poissons, modèle hydraulique, modèle biologique, couplage physique-biologique.

\section{MICROHABITAT METHODOLOGY : PRINCIPLES AND PROCEDURES.}

\begin{abstract}
In this note, we present, on potential users request, the microhabitat methodology procedures developed by CEMAGREF since 1985 and adapted from IFIM (US Fish and Wildlife Service). The models principles involved in this methodology are exposed. Measurement procedures are explained in the light of the hydraulic model principle and of the crossing procedure of biological and hydraulic informations. Application limits are exposed.
\end{abstract}

Key-words : «microhabitat» methodology procedures (IFIM), fish habitat, hydraulic model, biological model, physical-biological crossing procedure.

\section{INTRODUCTION}

\section{Intérêt de l'étude des déterminants physiques de l'habitat aquatique}

L'hydrodynamique des cours d'eau constitue le cadre physique au sein duquel se produisent les processus biologiques et s'organise le monde vivant. Les conditions physiques de l'habitat déterminent la structure des peuplements (SCHLOSSER, 1982 ; BAIN et al., 1988) ; il est donc indispensable de comprendre et d'évaluer le rôle de l'habitat dans le fonctionnement écologique des cours d'eau. L'utilisation anthropique de la ressource en eau se concrétise souvent par une modification du régime hydrologique, et donc des conditions d'habitat physique. Le développement d'outils est donc nécessaire pour quantifier l'habitat et les impacts de la gestion des débits sur la faune piscicole. 


\section{Outils développés}

II existe plusieurs méthodologies pour la détermination des débits réservés (revue de REISER et al., 1989) visant à relier les caractéristiques physiques de l'habitat aux caractéristiques biologiques des peuplements. La principale méthode utilisée pour les populations de poissons est la méthode des microhabitats inspirée par WATERS (1976), puis mise au point aux Etats-Unis sous la dénomination de alnstream Flow Incremental Methodology" ou IFIM (US Fish and Wildlife Service, Fort Collins, BOVEE, 1982). La méthodologie PHABSIM (Physical HABitat SIMulation) constitue la partie "Habitat» incluse dans IFIM. Elle permet de prédire la quantité d'habitat favorable au poisson, dans une portion de cours d'eau, en fonction de différents paramètres hydrodynamiques (MILHOUS et al., 1989 ; STALNAKER et al., 1989).

Depuis 1985, une méthode semblable, adaptée aux cours d'eau français, est développée par le Laboratoire d'Hydroécologie Quantitative du CEMAGREF de Lyon (SOUCHON et al., 1989). Elle est principalement destinée à fournir une aide à la détermination des débits réservés en aval des barrages, dans l'esprit de la loi relative à la pêche en eau douce de 1984 (article L.232-5 du Code rural). Elle peut également servir d'outil de recherche pour évaluer une sensibilité biologique des cours d'eau aux variations de l'habitat physique.

\section{Principe de la méthode des microhabitats}

La méthode des microhabitats fournit une quantification des capacités d'accueil potentielles pour le poisson, en fonction du débit. Dans ses grands principes, elle consiste à déterminer, sur une portion de cours d'eau, la surface d'habitat favorable à une espèce en couplant :

- d'une part, les composantes de la structure physique d'habitat. Les variables morphodynamiques retenues sont la Hauteur d'eau, la Vitesse du courant et la granulométrie du Substrat (HVS). Elles sont reconstituées, pour différents débits, au moyen d'un modèle hydraulique renseigné à partir d'une description hydraulique et topographique de la station étudiée.

- d'autre part, un modèle biologique traduisant les relations entre la densité relative des différents stades de développement des espèces de poisson et les valeurs des variables prises en compte dans le modèle hydraulique. Les relations habitats-poissons sont traduites en courbes de préférence.

Nous présentons ici les principes de mise en œuvre et les limites d'application de la méthode des microhabitats adaptée par le Laboratoire d'Hydroécologie Quantitative du CEMAGREF de Lyon.

\section{PRINCIPES DE FONCTIONNEMENT ET DE MISE EN CEUVRE}

La démarche comporte quatre étapes principales (fig. 1a et 1b) qui s'articulent autour des principes de fonctionnement des modèles hydrauliques et biologiques.

\section{Modélisation et mesures des variables physiques}

\section{Modélisation hydraulique}

Les estimations de l'évolution des paramètres hydrauliques en fonction du débit sont réalisées à partir d'un modèle hydraulique monodimensionnel en écoulement permanent, avec redistribution latérale des vitesses, adapté d'aprés le logiciel FLUVIA (BAUME et POIRSON, 1984 ; TROCHERIE, 1987). Le modèle calcule la ligne d'eau en régime fluvial. Dans ce cas, la ligne d'eau sur une portion du cours d'eau dépend, outre des conditions morphologiques locales (profil en travers, pente, rugosité du substrat) et du débit, du tirant d'eau à l'aval de cette portion. Le contrôle de la ligne d'eau est alors dit aval.

Pour entreprendre la modélisation, il est nécessaire de pouvoir calculer pour chaque débit le tirant d'eau sur une section située à l'aval de la portion. Le calcul est ensuite effectué de section en section, de l'aval vers l'amont. Le choix de la section aval représente 
Bull. Fr. Pêche Piscic. (1995) $336: 41-54 \quad-43-$

\section{1- MACRODESCRIPTION DU COURS D'EAU}

sectorisation, cartographie des faciès, choix de stations représentatives

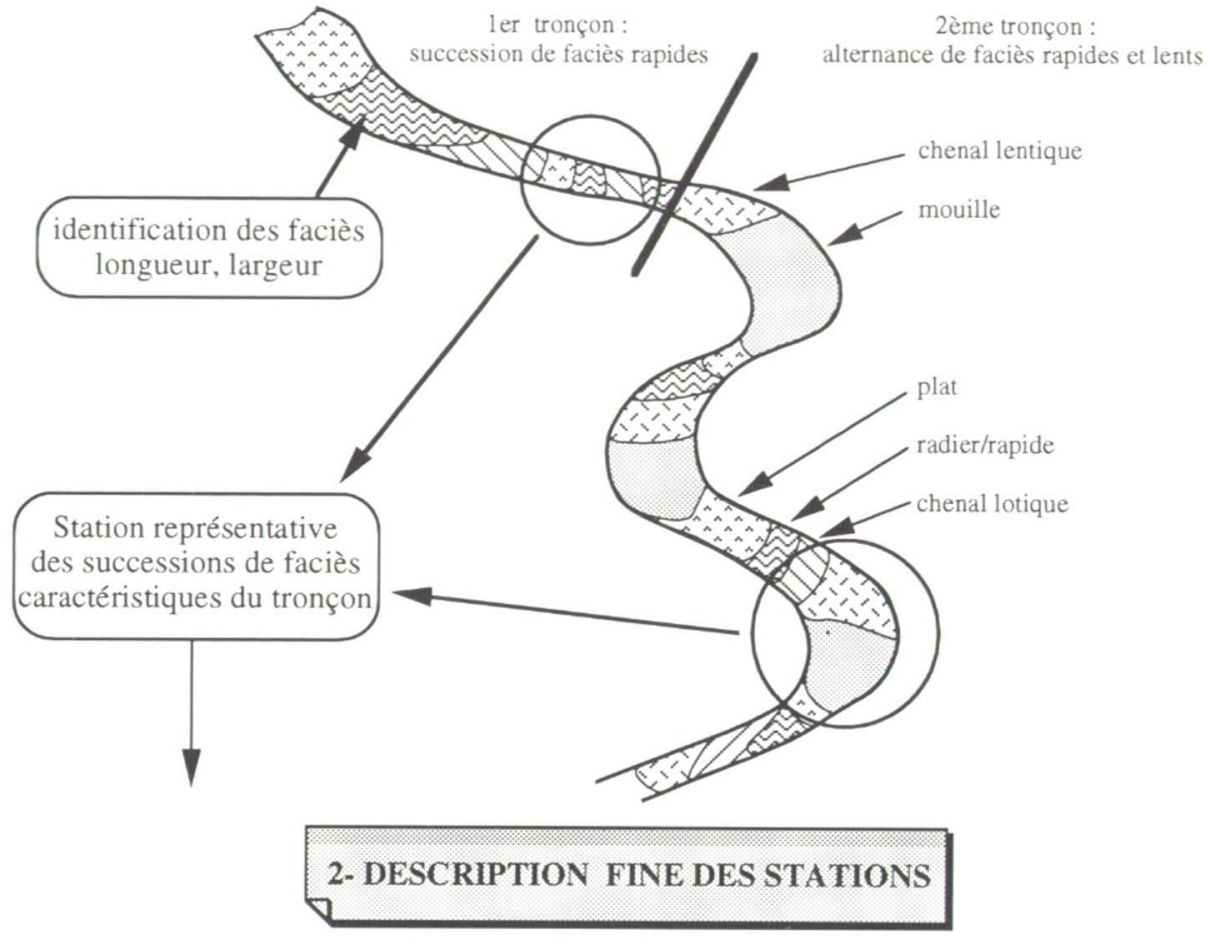

description topographique et hydraulique des stations (en moyenne 3 transects perpendiculaires à l'écoulement par faciès)
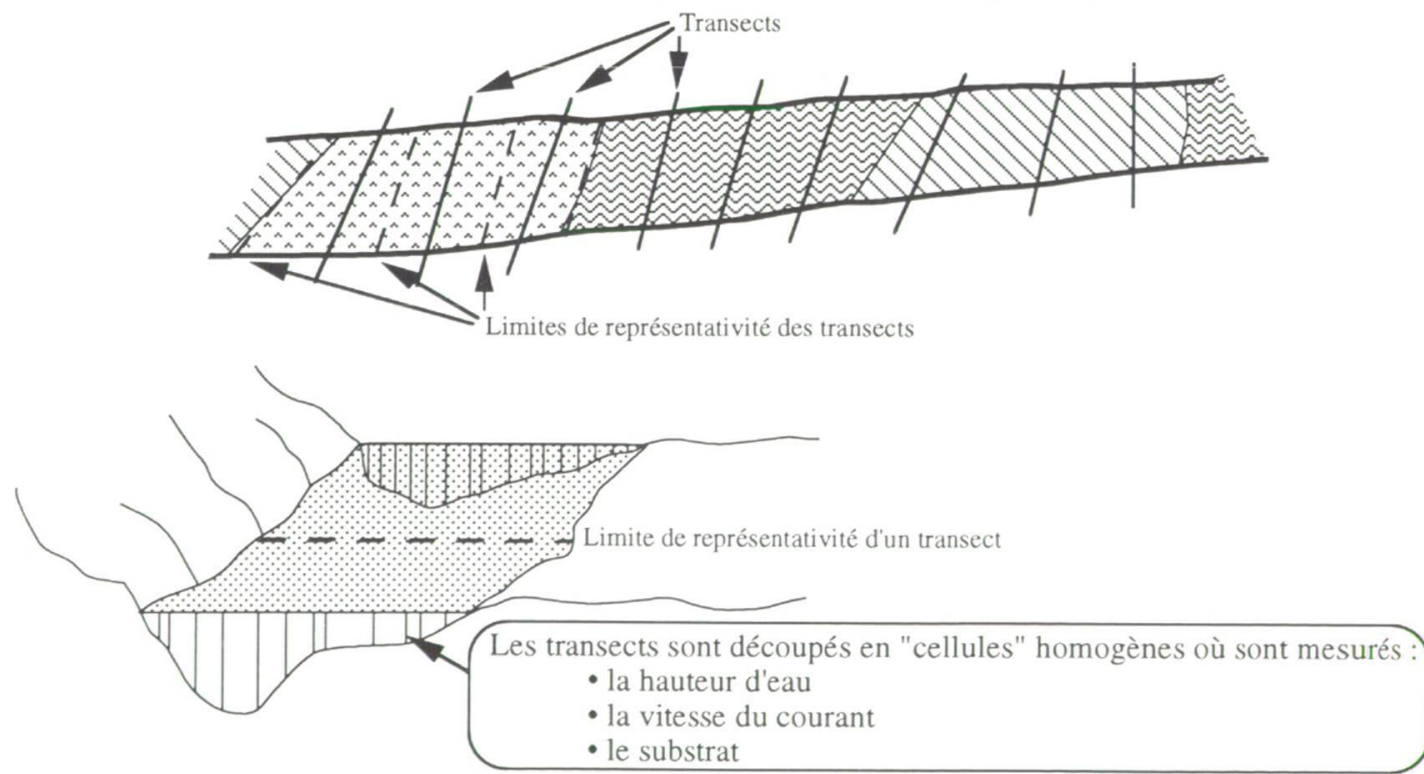

Figure 1a : Schéma de principe de la méthode des microhabitats utilisée par le CEMAGREF. Protocoles de description et de prises de mesures.

Figure 1a : Scheme of CEMAGREF microhabitat methodology principle. Physical description and hydraulic measurement procedures. 


\section{3- COUPLAGE PHYSIQUE - BIOLOGIQUE}

modélisation hydraulique et couplage avec un modèle biologique

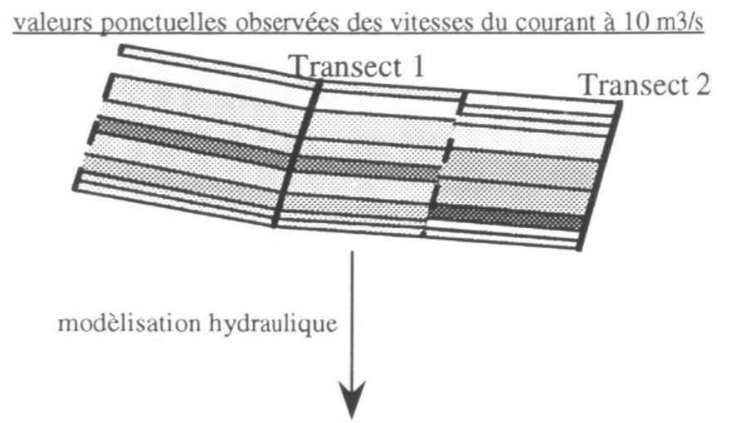

valeurs ponctuelles simulées des vitesses du courant à $20 \mathrm{~m} 3 / \mathrm{s}$

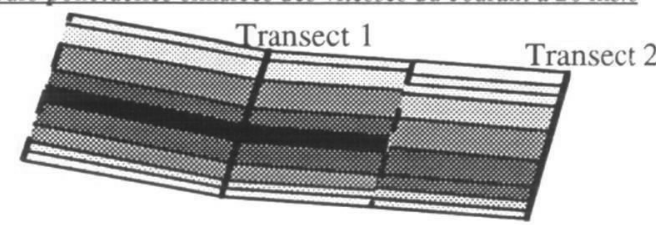

\section{Débit modélisé :}

un modèle hydraulique recalcule

pour chaque cellule

- la hauteur d'eau

- la vitesse du courant

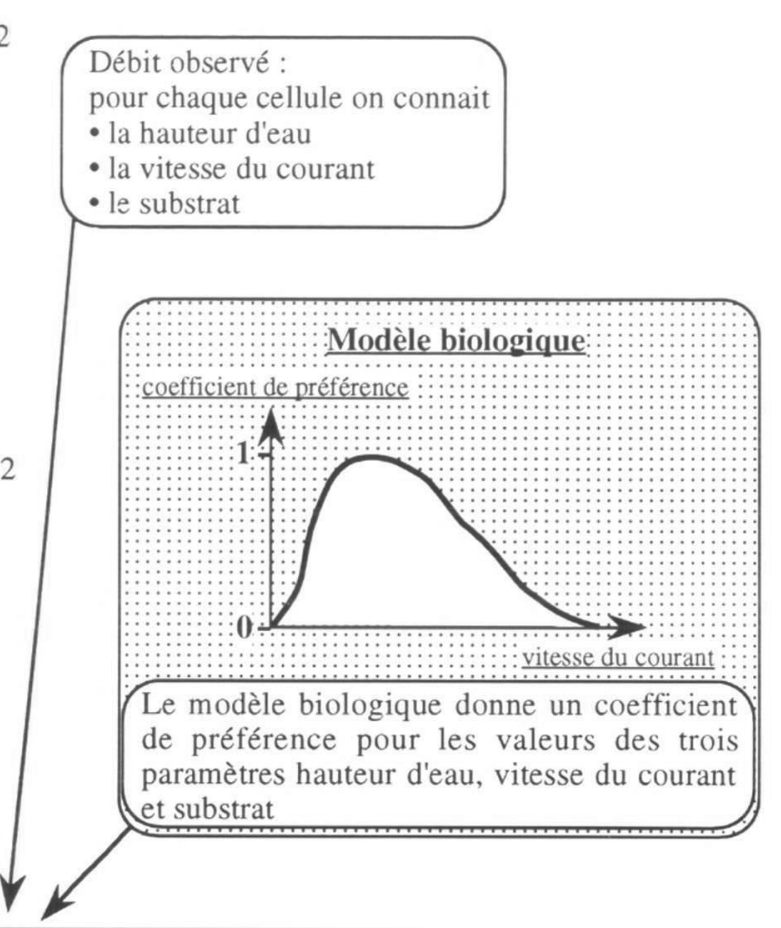

calcul de valeur d'habitat pour les débits observés et simulés

- par faciès

- par station

- extrapolation à tout le tronçon

\section{AIDE A L,A DECISION}

choix du débit à réserver : négociations en fonction des objectifs retenus pour le site

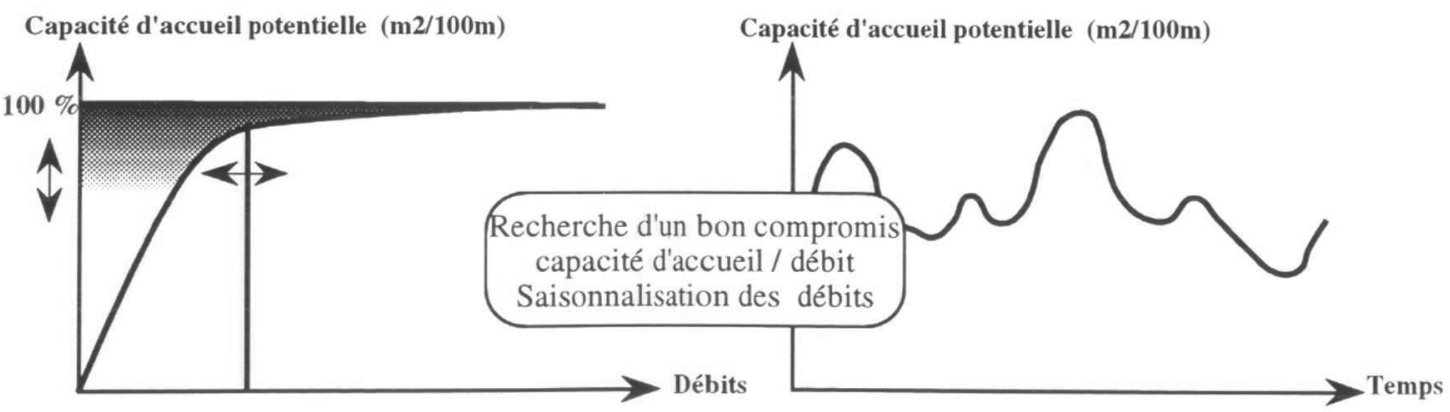

Figure 1b: Schéma de principe de la méthode des microhabitats utilisée par le CEMAGREF. Protocoles de simulation et d'aide à la détermination des débits réservés.

Figure 1b : Scheme of CEMAGREF microhabitat methodology principle. Simulation procedure and instream flow decision tool. 
un point clé de la modélisation. II est important que le tirant d'eau de cette section soit indépendant des conditions aval même à fort débit (fig. 2). En régime fluvial, une telle section peut être située soit dans un long faciès lotique (plat, chenal lotique, avec un tirant d'eau supérieur à la taille des éléments du substrat) de pente et de section constantes (fig. 2a), soit au niveau d'une rupture de pente nette (passage d'un écoulement de type fluvial avec une surface libre lisse à un écoulement torrentiel, fig. 2b) ou d'un seuil dénoyé (fig. 2c).

Dans les deux cas, des lois hydrauliques permettent de calculer le tirant d'eau en fonction du débit. Le fonctionnement du modèle hydraulique est présenté en annexe. Le modèle actuel repose sur la loi hydraulique correspondant au long faciès lotique (fig. 2a). Ce choix vient du fait que ce type de section est plus facile à trouver dans une rivière à morphologie naturelle.

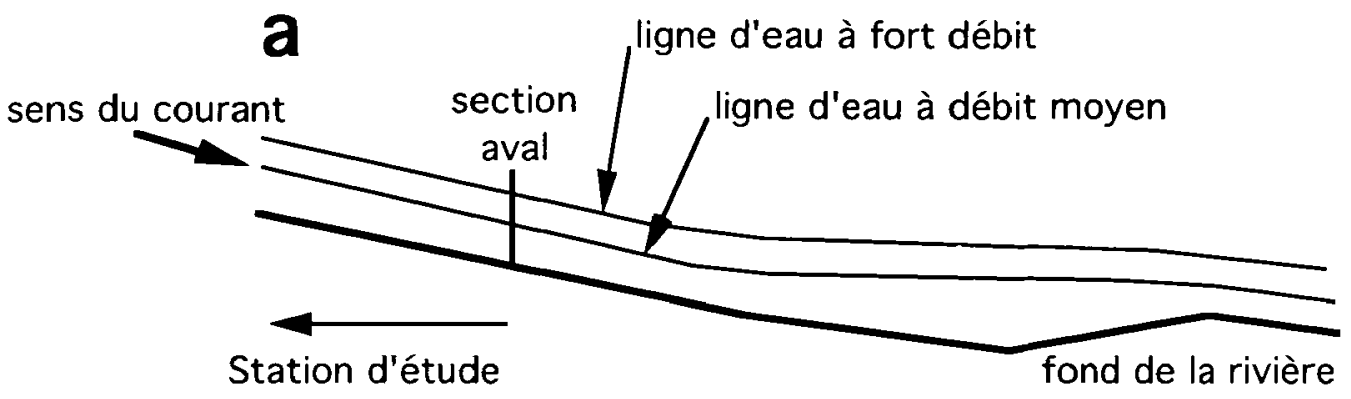

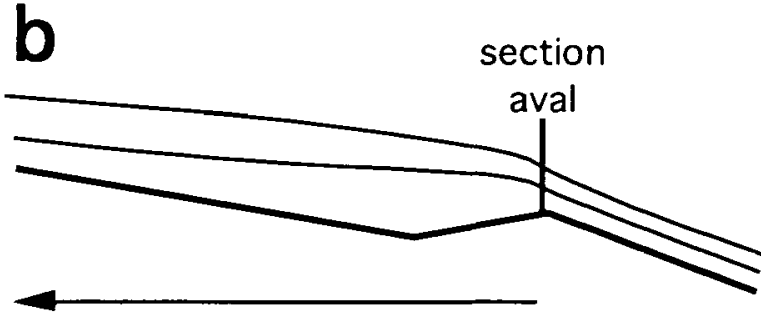

Station d'étude

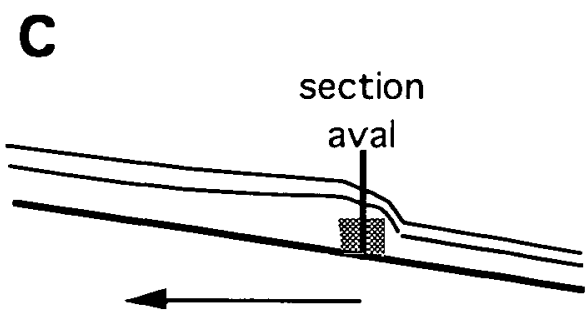

Station d'étude

Figure 2 : Schéma du profil en long d'un cours d'eau. Exemples de sections aval : a- section aval sur un long faciès lotique.

b- section aval sur une rupture de pente.

c- section aval sur un seuil.

Figure 2 : Scheme of river length profiles. Downstream sections examples :

a- downstream section on a long and lotic morphological feature.

b- downstream section on a slope rupture.

c- downstream section on a weir.

\section{Protocole de mesures}

La méthode fait l'objet d'un protocole de mesures précis (MALAVOI et SOUCHON, 1989), dans un double souci de représentativité physique et biologique de l'habitat.

\section{Sectorisation des tronçons et choix des stations}

Le choix des stations s'effectue après une description des faciès d'écoulement du cours d'eau étudié selon la définition de MALAVOI (1989), qui constitue l'étape de "sectorisation" (fig. 1a, étape 1). Elles doivent être représentatives de la diversité des faciès d'écoulement des tronçons décrits dans cette étape. Elles sont en outre choisies pour pouvoir 
ensuite pratiquer des échantillonnages biologiques (invertébrés benthiques et poissons). Elles doivent donc avoir une taille et une diversité suffisantes pour que les différents stades et les différentes espèces présentes dans le tronçon puissent être échantillonnées.

Pour satisfaire à ce double objectif (représentativité morphologique et biologique), les stations contiennent généralement une à deux successions de faciès contigus (séquences au sens de MALAVOI, 1989) rencontrés dans la sectorisation. La longueur d'une station est généralement de l'ordre de 10 à 14 fois la largeur du lit mouillé à un débit moyen. Pour représenter correctement un tronçon de rivière, on conseille généralement de choisir deux stations, soit trois ou quatre séquences de faciès. Rappelons également que les stations doivent impérativement comporter une section aval afin que les lois servant de point de départ au modèle hydraulique puissent être appliquées (ct. § précédent).

\section{Description des stations (fig. 1a, étape 2)}

Les stations sont découpées en segments homogènes pour les écoulements et les mosaïques de substrat qui les composent. Ce découpage rend compte de l'hétérogénéité longitudinale de la station. Un faciès est généralement découpé en trois segments homogènes (un en tête, un au centre, et un en queue), mais peut varier de deux à quatre selon les cas. Chaque segment est représenté par une section ou transect, matérialisé par un multimètre à ruban tendu perpendiculairement à l'écoulement. Les mesures prises au long des transects seront extrapolées de part et d'autre du transect, jusqu'aux limites du segment considéré, avec l'hypothèse que les conditions relevées au niveau du transect sont représentatives de tout ce segment. La limite entre deux segments est appelée limite de représentativité. La limite aval d'un segment forme la limite amont du segment suivant. Du point de vue biologique, la station est donc vue comme une succession de segments homogènes représentés par des transects.

Le choix de l'emplacement des transects est également fondamental pour un bon fonctionnement du modèle hydraulique. En effet, l'image de la station utilisée par le modèle hydraulique ne s'appuie que sur les sections reliées entre elles pour créer le profil longitudinal. Les ruptures de pente devront donc être correctement représentées par ces sections.

\section{Relevé topographique}

Le relevé topographique consiste à localiser dans l'espace les transects ainsi que leurs limites de représentativité. Une lunette à niveau et une mire positionnée en différents points fournissent les renseignements nécessaires (fig. 3). Ce relevé offre une visualisation cartographique de la station (vues en plan et profils en travers), un calcul de toutes les surfaces décrites (cellules, faciès, station) et un profil longitudinal avec les cotes de la surface libre et du fond. Le substrat et la topographie des berges sont également décrits afin d'obtenir des profils en travers suffisamment larges pour permettre la modélisation hydraulique à des débits plus forts.

\section{Mesures hydrauliques et granulométriques (fig. 1a, étape 2)}

Sur chaque transect, on décrit, pour le débit observé, les conditions d'écoulement dans le lit mouillé. On définit des surfaces élémentaires ou "cellules" s'étendant entre les limites de représentativité. Leur largeur dépend de l'hétérogénéité latérale rencontrée : tout changement notable d'un des trois paramètres HVS définit une nouvelle cellule. En pratique, la largeur des cellules varie de quelques centimètres à un mètre dans les cas les plus homogènes. Chaque cellule est caractérisée à partir de points de mesures (fig. 4) où on relève:

- la hauteur d'eau $(H)$, pouvant prendre une valeur négative lorsque le point est hors d'eau (blocs ou rochers émergents),

- la vitesse du courant (V), mesurée à l'aide d'un micro-moulinet ou d'un courantomètre électromagnétique, à $0,2,0,4$ et 0,8 de la hauteur d'eau à partir du fond. La vitesse moyenne est obtenue par la formule suivante, d'après une NORME ISO (1983) : Vmoy $=1 / 4\left(V_{0,2}\right)+1 / 2\left(V_{0,4}\right)+1 / 4\left(V_{0,8}\right)$. Lorsque la hauteur d'eau est inférieure à $20 \mathrm{~cm}$, seule la vitesse à $0,4^{\prime} \mathrm{H}$ est relevée. Elle est considérée comme proche de la vitesse moyenne, 


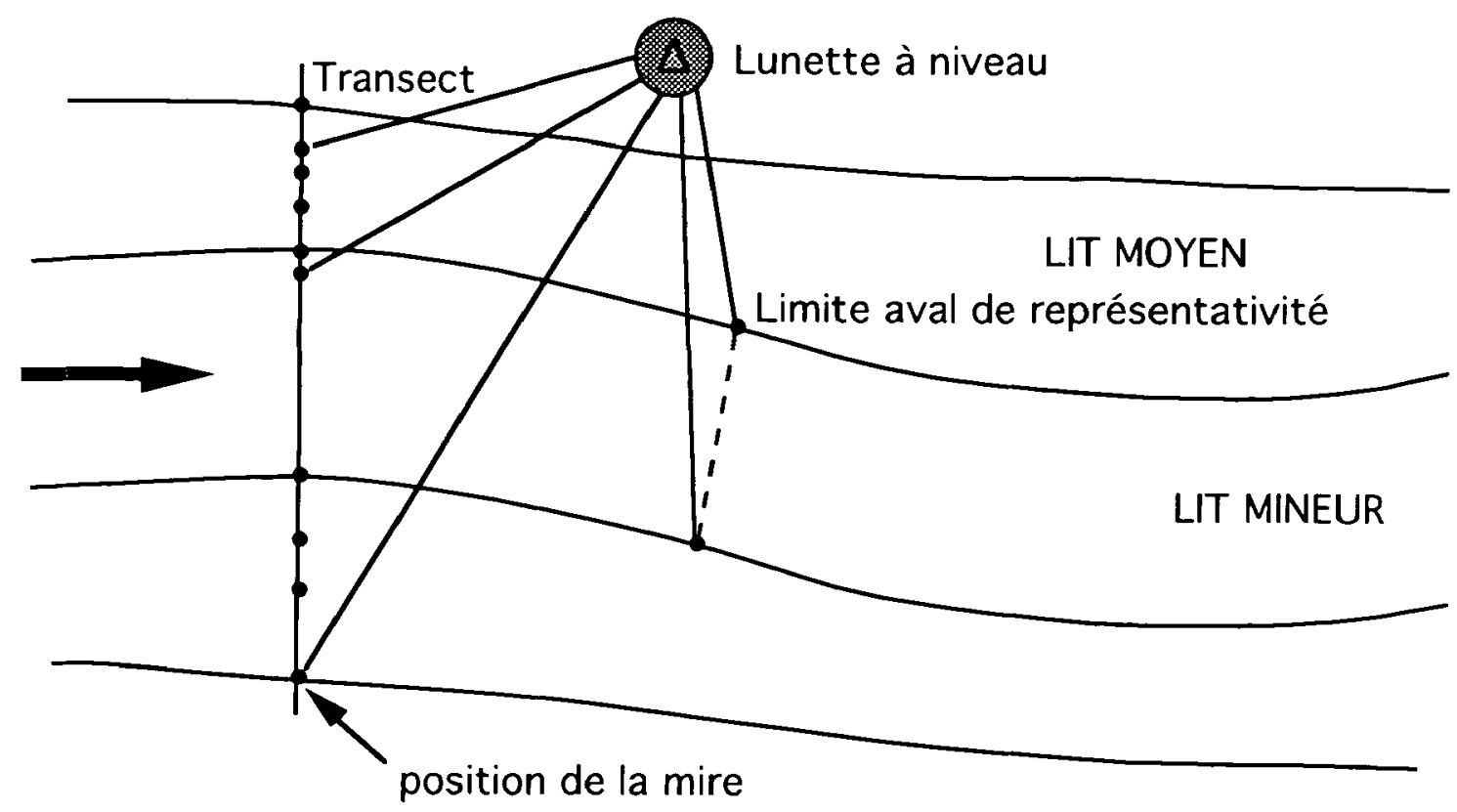

Figure 3 : Protocole de description topographique (MALAVOI et SOUCHON, 1989). Figure 3 : Topographic description procedure (MALAVOI and SOUCHON, 1989).

- le substrat (S), décrit pour chaque cellule selon un code établi par MALAVOI (1989) à partir de l'échelle granulométrique de Wentworth modifiée. Ce code comporte trois champs qui permettent de décrire l'agencement des différentes fractions granulométriques (MALAVOI et SOUCHON, 1989) : la plus grossière (GR) dès lors qu'elle couvre plus de $10 \%$ de la cellule, la première fraction dominante (DOM1) et la deuxième fraction dominante s'il y a lieu (DOM2, couvrant au moins $30 \%$ de la surface). Un substrat colmaté où le dépôt limoneux recouvre complètement les éléments grossiers sous-jacents doit être décrit comme un substrat totalement limoneux.

\section{Champ d'utilisation du modèle}

Le modèle est performant dans une gamme de débits simulés environ 10 fois supérieurs et 5 fois inférieurs au débit observé. Au-delà de cette gamme, il risque de diverger par rapport à la réalité (l'incertitude est alors difficilement calculable) ou d'atteindre des limites de validité du modèle (hauteur d'eau inférieure à la taille des éléments du substrat ou nombreuses sections en écoulement torrentiel). Ces limites peuvent cependant dépendre du type de cours d'eau : par exemple, dans un cours d'eau à granulométrie grossière, si le débit simulé est faible, on peut obtenir des hauteurs d'eau trop faibles par rapport à la granulométrie et les calculs deviennent irréalisables.

\section{Modèle biologique}

Les modèles biologiques les plus largement répandus, pour l'application de la méthode des microhabitats, se présentent sous la forme de courbes de préférence (ou profils écologiques) pour un paramètre physique. La représentativité de ces courbes est un déterminant essentiel de la qualité de ces outils de prédiction. Localement, la dynamique saisonnière d'utilisation de l'habitat par les poissons peut être reliée à une variation de la disponibilité en habitat (GROSSMAN et al., 1987). Pour cette raison, on distingue deux types de courbes: 


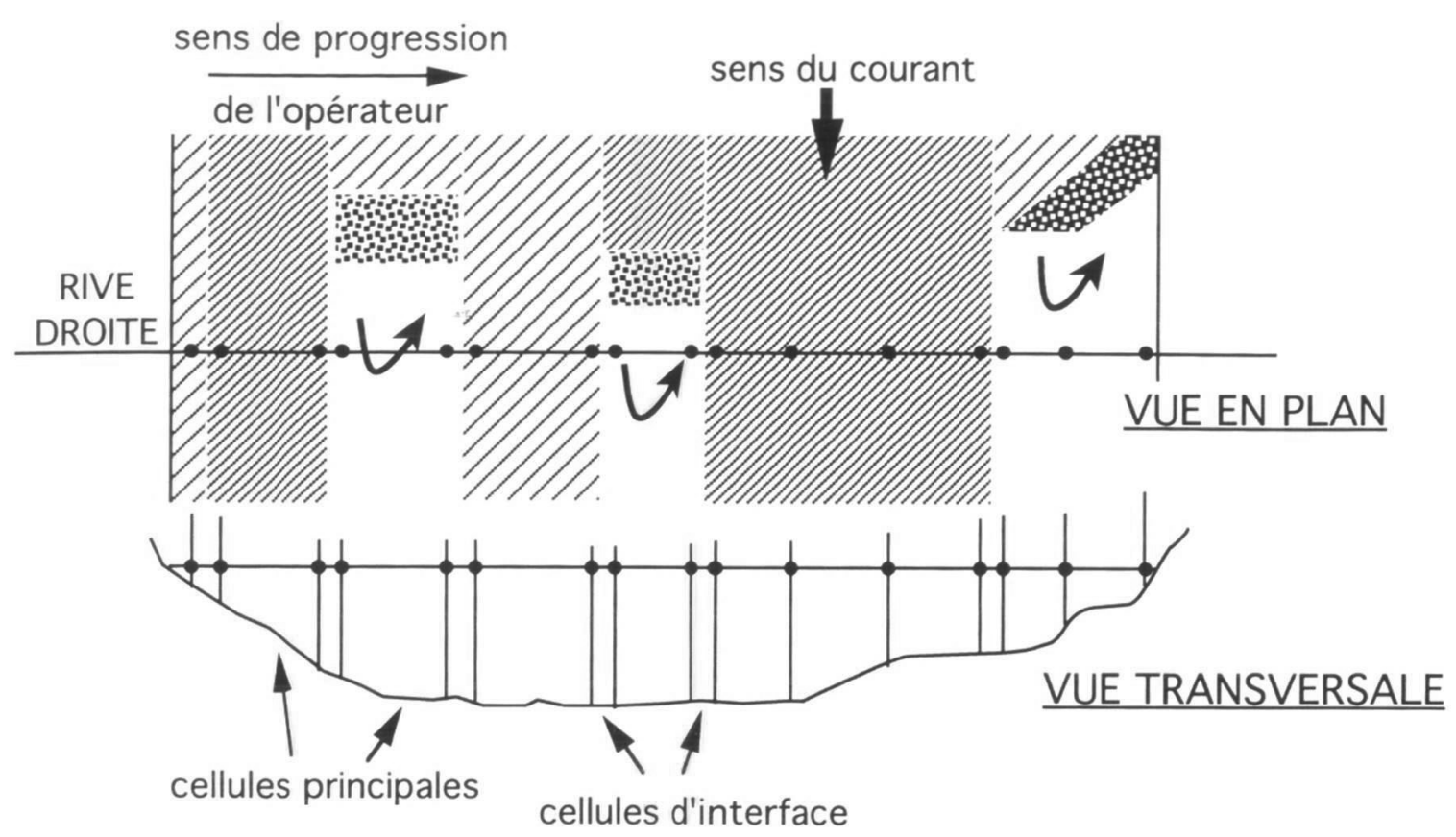

\begin{tabular}{|ll|}
\hline - point de mesure & U contre-courant \\
II//, écoulement lent & bloc \\
WIII///, écoulement rapide & embâcle \\
\hline
\end{tabular}

Figure 4 : Protocole de description des cellules et des points de mesures hydrauliques (MALAVOI et SOUCHON, 1989).

Figure 4 : Cells description and hydraulic measurements procedure (MALAVOI and SOUCHON, 1989).

- les courbes d'utilisation construites à partir de données de terrain relatives à un site et non. pondérées par la disponibilité en habitat. Ces courbes sont donc spécifiques à un site.

- les courbes de préférence plus générales, pondérées par la disponibilité en habitat ou issues des connaissances résumées dans la littérature. Ces courbes sont établies soit par synthèse bibliographique, soit par enquêtes auprès d'experts ou encore à partir de nombreuses mesures directes (BOVEE, 1986). Elles expriment les préférences (codées de 0 à 1) des stades ontogéniques d'une espèce pour les trois variables d'habitat (Hauteur, Vitesse, Substrat) considérées comme prépondérantes dans la sélection des habitats par les poissons (GORMAN et KARR, 1978 ; GROSSMAN et al., 1987 ; SCHLOSSER, 1987). Elles permettent de prédire la répartition théorique des poissons en fonction des variables physiques.

Dans un cadre général d'application de la méthode des microhabitats, nous préconisons les courbes de préférence qui ont une signification plus large, surtout lorsqu'elles bénéficient d'une validation et d'un retour d'expérience sur de nombreux sites.

Plusieurs courbes d'utilisation ont été établies en France pour la truite fario (Salmo trutta fario, L. 1758) (BELAUD et al., 1989). Les courbes de préférence actuellement utilisées dans la méthode des microhabitats ont été testées et validées dans différentes écorégions (Jura sud, Alpes sud, Préalpes, Massif Central) pour la truite fario (FRAGNOUD, 1987 ; SOUCHON et al., 1989). 
Des modèles biologiques pour d'autres espèces sont aussi en cours de validation: Barbeau (Barbus barbus, L. 1758) (POUILLY et SOUCHON, 1994), Ablette (Alburnus alburnus, L. 1758), Blageon (Leuciscus soufia, Risso 1826), Chevaine (Leuciscus cephalus, L. 1758), Gardon (Rutilus rutilus, L. 1758), Goujon (Gobio gobio, L. 1758), Loche franche (Noemacheilus barbatulus, L. 1758), Perche fluviatile (Perca fluviatilis, L. 1758), Spirlin (Alburnoides bipunctatus, Bloch 1782), Vairon (Phoxinus phoxinus, L. 1758) (POUILLY, 1994).

\section{Couplage des modèles physique et biologique (fig. 1b, étape 3)}

Les paramètres hydrauliques observés ou calculés par le modèle hydraulique sont utilisés dans le calcul des surfaces d'habitat potentiellement utilisables par le poisson. Ces surfaces sont appelées Surfaces Pondérées Utiles (SPU), par rapport à leur mode d'obtention : la $S P U_{i}$ d'une cellule $n^{\circ} i$ est égale à la surface de cette cellule $\left(A_{j}\right)$ pondérée par le produit des coefficients de préférence lus sur les trois courbes de préférence $\left(P\left(H_{j}\right)\right.$, $P\left(V_{j}\right)$ et $P\left(S_{i}\right)$ ) pour les valeurs $H_{i}, V_{i}$ et $S_{j}$ de la cellule, à un débit donné. Les SPU des différentes cellules sont ensuite additionnées pour obtenir les SPU des transects, des faciès ou de toute la station: $S P U=\Sigma_{i} A_{i} \times P\left(H_{i}\right) \times P\left(V_{i}\right) \times P\left(S_{j}\right)$. Le coefficient $P\left(S_{i}\right)$ est calculé par la moyenne des indices de préférence obtenus pour les différentes fractions granulométriques décrites (GR, DOM1 et éventuellement DOM2), pondérées par les coefficients empiriques : respectivement $0,2,0,4$ et 0,4 ou 0,2 et 0,8 en l'absence de DOM2.

Ce modèle multiplicatif sous-entend que les trois paramètres ont une importance équivalente dans le choix de l'habitat. Le logiciel américain PHABSIM (MILHOUS et al., 1989) propose deux possibilités supplémentaires de calcul de la capacité d'accueil potentielle d'un habitat reposant sur des hypothèses biologiques différentes:

- un modèle qui pondère la surface des cellules par la moyenne géométrique des coefficients de préférence. Dans ce cas, l'hypothèse est l'existence d'un effet de compensation entre les variables : si deux des trois variables sont optimales, elles compenseront la troisième si elle est faible,

- un modèle qui pondère la surface des cellules par le coefficient de préférence le plus faible. On considère alors que la sélection des habitats se fait en fonction d'un facteur limitant.

Ces différents modèles conduisent à des résultats de capacité d'accueil différents pour une même station (GAN et MCMAHON, 1990). L'état actuel des connaissances ne permet pas de faire un choix objectif entre ces options. Un choix ouvert de ces options, comme le propose le logiciel PHABSIM (MILHOUS et al., 1989), laisse souvent l'utilisateur perplexe. Tant que les différents résultats n'ont pu être étayés par une validation adaptée au cas traité, notre choix se porte sur le seul modèle multiplicatif.

Afin de rendre les stations étudiées comparables, les SPU peuvent être ramenées à 100 mètres de linéaire de cours d'eau. Pour comparer des sites de largeurs différentes, on peut également ramener les SPU à une unité de surface. La capacité d'accueil s'exprime alors en valeur d'habitat (VH) égale au pourcentage de SPU par rapport à la surface mouillée (SM).

On obtient plusieurs outils d'interprétation (fig. 1b, étapes 3 et 4 ) : cartographies des variables physiques ou des valeurs d'habitat pour chaque cellule à un débit donné ; courbes de SPU en fonction du débit ; courbes de SPU en fonction du temps. Ces dernières sont réalisées à partir de chroniques de débit, elles permettent la prise en compte du régime hydrologique. Ces courbes font actuellement l'objet de recherches méthodologiques et de procédures de validation (CAPRA et al., 1995).

\section{Temps et moyens humains nécessaires pour une station}

Le temps nécessaire pour décrire une station comportant une douzaine de transects est variable en fonction de la taille du cours d'eau et de l'expérience des opérateurs. En moyenne, les mesures prennent une demi-journée à une journée pour six ou quatre personnes. Ce temps est donc équivalent à celui nécessaire pour réaliser une pêche 
électrique à deux passages sur une même station. Il est important de ne pas dépasser la journée afin de réduire les risques de subir des fluctuations du débit au cours de la prise de mesures rendant alors le calage hydraulique délicat.

Une ou deux personnes effectuent préalablement la sectorisation, le choix des stations et le marquage de l'emplacement des transects.

La réalisation du relevé topographique par deux personnes nécessite le même temps que les mesures hydrauliques réalisées par deux autres binômes.

Une journée est ensuite nécessaire à une personne pour saisir et traiter les données sur ordinateur. Ce travail est à présent facilité par la mise à disposition d'un logiciel de traitement des différentes informations : EVHA (EValuation de l'HAbitat) développé au Laboratoire d'Hydroécologie Quantitative, sous environnement PC/Windows (GINOT et SOUCHON, 1995).

\section{DISCUSSION}

La méthode des microhabitats fournit tout d'abord une bonne description de l'évolution des paramètres physiques en fonction du débit et permet, avant même le couplage avec le modèle biologique, d'obtenir des informations complémentaires à l'analyse biologique sur le fonctionnement physique et morphologique de la portion de cours d'eau étudiée.

L'estimation des capacités d'accueil théoriques pour le poisson apporte ensuite des éléments objectifs pour l'aide à la décision lors du choix d'un débit réservé, ou pour la description de l'évolution des conditions physiques de l'habitat des poissons.

\section{Limites de la méthode}

La méthode des microhabitats est principalement utilisée pour déterminer la quantité d'habitat disponible aux débits moyens ou faibles. Les populations de truite des petits cours d'eau sont limitées principalement par les événements hydrologiques les plus secs (BOVEE, 1982 ; SOUCHON et al., 1989 ; JOWETT, 1992). Des crues très fortes qui interviennent à des pas de temps moins fréquents peuvent également influencer la dynamique des peuplements.

La campagne de mesures hydrauliques et topographiques doit s'effectuer préférentiellement à débit moyen à faible afin d'obtenir des plages de débits modélisés correspondant aux événements hydrologiques le plus fréquemment limitants. Des recherches sont actuellement en cours pour améliorer la performance des modèles hydrauliques pour le calcul de la ligne d'eau et la redistribution latérale des vitesses dans le cas de portions de cours d'eau à faible tirant d'eau (E. HEROUIN, comm. pers. ; DUPASQUIER, 1994). De plus, le découpage en cellules pourrait être amélioré en remplaçant la géométrie rectangulaire par un découpage plus fin capable de détecter des obstacles ponctuels. Des travaux sont en cours pour adapter un mode de description triangulaire au sein d'un autre modèle hydraulique (LE COARER et DUMONT, 1995).

Les modèles biologiques utilisant des courbes de préférence ont fait l'objet de critiques (MATHUR et al., 1985 ; ORTH, 1987 ; SHIRVELL, 1989 ; SOUCHON et al., 1989) notamment sur le choix des variables physiques retenues (HVS) et sur l'utilisation monovariée de ces variables qui interviennent en réalité de façon combinée dans la sélection de l'habitat par les poissons. D'autre part, ces variables devraient être complétées par d'autres tout aussi importantes pour les poissons (abris, végétation) et analysées simultanément dans des modèles multivariés. De tels modèles sont en cours de développement (POUILLY et SOUCHON, 1994 ; POUILLY, 1994). Les abris ne sont que partiellement pris en compte par la granulométrie du substrat ; des travaux de recherches ont d'ores et déjà été entrepris pour mieux comprendre leur rôle dans la sélection de l'habitat par les poissons afin de pouvoir les inclure dans les modèles. 


\section{Contexte plus général}

Toute étude sur l'habitat physique doit toujours être replacée dans un contexte plus large tenant compte des autres compartiments de l'écosystème aquatique. Une bonne connaissance de la rivière étudiée est donc indispensable, notamment en ce qui concerne les peuplements en place et le régime hydrologique :

- les résultats de la méthode reflètent une situation potentielle qui correspondrait à un peuplement monospécifique et non influencé par d'autres paramètres limitants biotiques (prédation, compétition, ressources trophiques) ou abiotiques (température, physico-chimie),

- le découpage par transects s'avère imparfait pour décrire la répartition très locale des conditions favorables au frai. Dans ce cas, les valeurs de SPU résultantes pour ce stade peuvent paraître très faibles. D'autre part, l'échelle spatiale de sélection des habitats de reproduction est supérieure à celle d'une station d'étude. Les conclusions concernant la reproduction de l'espèce considérée doivent donc être accompagnées d'une cartographie précise des zones de reproduction potentielles sur la station et sur l'ensemble du tronçon étudié,

- les cours d'eau sont caractérisés par une variabilité des conditions de débit structurant les communautés, or les résultats des SPU en fonction du débit n'intégrent pas la variabilité du débit dans le temps. L'analyse des chroniques de SPU en fonction du temps illustre l'aspect limitant des périodes d'étiage. Différents scénarios de débit (année sèche ou "moyenne") doivent être testés pour déterminer les événements hydrologiques correspondant aux capacités d'accueil critiques pour les populations.

Tous ces éléments doivent être considérés dans l'interprétation finale des résultats. L'intégration de ces résultats dans un contexte plus général correspond d'autre part aux objectifs de gestion intégrée des écosystèmes définis dans la loi sur l'eau de 1992.

De plus, l'interprétation dépendra étroitement des objectifs de l'étude (débit réservé, débits variables, objectifs économiques, sauvegarde d'espèces à titre patrimonial,...). En effet, la première nécessité, avant l'utilisation de la méthode des microhabitats, est la détermination d'un objectif, explicite et précis, établi après concertation des organismes de protection du milieu naturel, des usagers et des gestionnaires de l'ouvrage. Cet objectif servira de guide pour une interprétation objective des résultats de l'étude et aboutira à une décision mieux acceptée (OSBORNE et al., 1993).

\section{REMERCIEMENTS}

L'élaboration et l'évolution de la méthode présentée ont bénéficié d'apports conséquents de F. TROCHERIE, J.R. MALAVOI et J.G. WASSON. Nos plus vifs remerciements s'adressent également à $E$. HEROUIN et à $P$. BREIL pour une relecture critique du manuscrit et de nombreuses précisions sur la partie hydraulique. Ces recherches ont pu aboutir grâce à l'aide financière du Ministère de l'Environnement (Direction de l'Eau et SRETIE), d'EDF (Direction des Etudes et Recherches) et de l'Agence de l'eau Rhône-Méditerranée-Corse.

\section{BIBLIOGRAPHIE}

BAIN M.B., FINN J.T., BOOKE H., 1988. Streamflow regulation and fish community structure. Ecology, 69 (2), 382-392.

BATHURST J.C., 1982. Flow resistance in boulder bed streams in HEY R.D., BATHURST J.C., THORNE C.R., Gravel-bed rivers, 443-465, John Wiley and Sons Ltd.

BAUME J.P., POIRSON M., 1984. Modélisation numérique d'un écoulement permanent dans un réseau hydraulique maillé à surface libre, en régime fluvial. La houille blanche, 1 (2), 95-100.

BELAUD A., CHAVEROCHE P., LIM P., SABATON C., 1989. Probability of use curves applied to brown trout (Salmo trutta L.) in river of Southern France. Reg. Riv. : Res. \& Manag., 3, 321-336. 
BOVEE K.D., 1982. A guide to stream habitat analysis using the Instream Flow Incremental Methodology. Instream Flow Information Paper $n^{\circ}$ 12, FWS/OBS 82/86, 248 p.

BOVEE K.D., 1986. Development and evaluation of habitat suitability criteria for use in the instream flow incremental methodology. Instream Flow Information Paper $n^{\circ} 21$, FWS/OBS 86/7, $188 p$.

CAPRA H., BREIL P., SOUCHON Y., 1995. A new tool to interpret magnitude and duration of fish habitat variations. Reg. Riv. : Res. \& Manag., 10, 281-289.

DUPASQUIER B., 1994. Détermination d'une loi de débit sur seuil naturel. Rapport de DEA ENGEES - Université L. Pasteur, Strasbourg, 105 p.

FRAGNOUD E., 1987. Préférences d'habitat de la Truite fario (Salmo trutta fario L.,1758) en rivière (quelques cours d'eau du sud-est de la France). Thèse Univ. Lyon I, CEMAGREF Lyon, $435 \mathrm{p}$.

GAN K., MC MAHON T., 1990. Variability of results from the use of PHABSIM in estimating habitat area. Reg. Riv. : Res. \& Manag., 5, 233-239.

GINOT V., SOUCHON Y., 1995. Logiciel EVHA. Evaluation de l'habitat physique des poissons en rivière (version 1.0, beta test). Vol. 1. Guide méthodologique $106 \mathrm{p}$. Vol. 2. Guide de l'utilisateur 66 p. Cemagref BEA/LHQ Lyon, France, et Ministère de l'Environnement, Dir. de l'Eau, Paris, France.

GORMAN O.T., KARR J.R., 1978. Habitat structure and stream fish communities. Ecology, 59, 507-515.

GROSSMAN G.D., DE SOSTOA A., FREEMAN M.C., LOBON-CERVIA J., 1987. Microhabitat use in a Mediterranean riverine fish assemblage. Oecol., 73, 490-500.

HEY H.D., 1982. Design equations for mobile gravel-bed rivers in HEY R.D., BATHURST J.C., THORNE C.R., Gravel-bed rivers, 553-580, John Wiley and Sons Ltd.

JARRET R.D., 1984. Hydraulics of high-gradient streams. J. Hydr. Eng., 110 (11), 1519-1539.

JOWETT I.G., 1992. Models of the abundance of large brown trout in New Zealand rivers. North American Journal of Fisheries Management, 12, 417-432.

LE COARER Y., DUMONT B., 1995. Modélisation de la morphodynamique fluviale pour la recherche des relations habitat-faune aquatique. In: Symposium "Relations entre le poisson et son habitat", Lyon, 6-8 Décembre 1994, in press.

MALAVOI J.R., 1989. Typologie des faciès d'écoulement ou unités morphodynamiques des cours d'eau à haute énergie. Bull. Fr. Pêche Piscic., 315, 189-210.

MALAVOI J.R., 1991. Méthodologie de sectorisation et de description des cours d'eau à pente forte et moyenne : application à une gestion intégrée des écosystèmes d'eau courante. Thèse Univ. Lyon III, CEMAGREF Lyon, $517 \mathrm{p}$.

MALAVOI J.R., SOUCHON Y., 1989. Méthodologie de description et quantification des variables morphodynamiques d'un cours d'eau à fond caillouteux. Exemple d'une station sur la Filière (Haute-Savoie). Rev. géo. Lyon, 64 (4), 252-259.

MATHUR D., BASON W.H., PURDY E.J., SILVER C.A., 1985. A critique of the Instream Flow Incremental Methodology. Can. J. Fish. Aqua. Sci., 42, 825-831.

MILHOUS R.T., UPDIKE M.A., SCHNEIDER D.M., 1989. Physical habitat simulation system reference manual - version 11. Instream Flow Information Paper $n^{\circ} 26$, Western Energy and Land Use Team, U.S. Fish and Wildlife Service, Ft Collins, Colorado, FWS/OBS 89/16, 216 p.

NORME ISO 748-1979 (F), 1983. Méthodes d'exploration du champ des vitesses in Organisation internationale de normalisation, Mesure de débit des liquides dans les canaux découverts, 57-80, Genève, Suisse.

ORTH D.J., 1987. Ecological considerations in the development and application of instream flow habitat models. Reg. Riv. : Res. \& Manag., 1, 171-181. 
OSBORNE L.L., BAYLEY P.B., HIGLER L.W., 1993. Lowland stream restoration : theory and pratice. Fresh. Biol., 29 (2), 187-342.

POUILLY M., 1994. Relations entre l'habitat physique et les poissons des zones à cyprinidés rhéophiles dans trois cours d'eau du bassin rhodanien : vers une simulation de la capacité d'accueil pour les peuplements. Thèse Univ. Lyon I / CEMAGREF Lyon, $260 \mathrm{p}$.

POUILLY M., SOUCHON Y., 1994. Simulation de l'habitat physique du barbeau fluviatile (Barbus barbus, L. 1758) : choix des modèles biologiques et sensibilité de la réponse. Bull. Fr. Pêche Piscic., 334, 213-225.

REISER D.W., WESCHE T.A., ESTES C., 1989. Status of instream flow legislation and practices in North America. Fisheries, 14 (2), 22-29.

SCHLOSSER I.J., 1982. Fish community structure and function along two habitat gradients in a headwater stream. Ecological Monographs, 52 (4), 395-414.

SCHLOSSER I.J., 1987. A conceptual framework for fish communities in small warmwater streams in Matthews W.J. and Heins D.C., Community and Evolutionary Ecology of North American Stream Fishes, University of Oklahoma Press, Norman, 17-24.

SHIRVELL C.S., 1989. Ability of Phabsim to predict chinook salmon spawning habitat. Reg. Riv. : Res. \& Manag., 3, 277-289.

SOUCHON Y., TROCHERIE F., FRAGNOUD E., LACOMBE C., 1989. Les modèles numériques des microhabitats des poissons : application et nouveaux développements. Rev. Sci. Eau, 2, 807-830.

STALNAKER C.B., MILHOUS R.T., BOVEE K.D., 1989. Hydrology and hydraulics applied to fishery management in large rivers. Can. Spec. Publ. Fish. Aqua. Sci., 106, 13-30.

TROCHERIE F., 1987. Mise en application de l'article 410 du Code rural. Elément d'une démarche de détermination des débits réservés. CEMAGREF / DPN, 85-15, 30 p.

WATERS B.F., 1976. A methodology for evaluating the effects of different streamflows on salmonid habitat in Orsborn J.F. and Allman C.H., Instream Flow Needs, American Fisheries Society, Western Division, Bethesda, Maryland, 254-266.

\section{ANNEXE}

\section{FONCTIONNEMENT DU MODĖLE HYDRAULIQUE}

\section{Equations de perte de charge}

Le modèle utilisé est détaillé par TROCHERIE (1987). Il est issu du logiciel "FLUVIA» de BAUME et POIRSON (1984). Ce modèle comporte des formules reliant la perte de charge à un coefficient de rugosité et aux paramètres hydrodynamiques (hauteur d'eau, vitesse du courant, largeur ou périmètre mouillé). Généralement, la formule utilisée est celle de MANNING-STRICKLER :

$$
J=V^{2} /\left(K_{s}^{2} * R^{4 / 3}\right)
$$

avec $J=$ perte de charge linéaire

$\mathrm{V}=$ vitesse moyenne du courant sur la section

$\mathrm{K}_{\mathrm{S}}=$ coefficient de rugosité

$\mathrm{R}=$ rayon hydraulique (section mouillée / périmètre mouillé).

La formule de MANNING-STRICKLER, bien adaptée aux cours d'eau à faible énergie (cours d'eau de plaine), est moins performante dans les cours d'eau à forte et moyenne énergie (pente élevée), où la rugosité du substrat est du même ordre de grandeur que la hauteur d'eau (TROCHERIE, 1987). A la place de cette équation, TROCHERIE (1987) préconise l'utilisation de la formule de LIMERINOS validée par 
plusieurs auteurs (BATHURST, 1982 ; HEY, 1982 ; JARRET, 1984). Cette formule exprime la perte de charge linéaire $(\mathrm{J})$ en fonction de la vitesse $(V)$, du rayon hydraulique $(R)$, de la profondeur maximale de la section $(\mathrm{hm})$, de la constante de gravité $(\mathrm{g})$ et de la rugosité représentée par le $\mathrm{D} 84$, défini comme la taille maximale théorique de l'axe b de $84 \%$ des éléments du substrat :

$$
J=\frac{v^{2}}{33 g R[\log 10(3,17 R / D 84)-0,314 \log 10(R / h m)]}
$$

Par hypothèse dans ce type de modèle, les coefficients de rugosité (le D84 pour la formule de LIMERINOS) sont considérés constants quel que soit le débit.

\section{Principe de modélisation}

\section{Sur la section aval}

Le modèle actuel permet uniquement le calcul pour une section aval située dans un long faciès lotique (plat, chenal lotique, avec un tirant d'eau supérieur à la taille des éléments du substrat) de pente et de section constantes. Le modèle peut être appliqué dans le cas d'une section aval située sur un seuil en béton, l'erreur théorique restant modérée (de l'ordre de $10 \%$ pour un débit 10 fois supérieur au débit observé, E. HEROUIN, comm. pers.).

La formule de Limerinos est utilisée pour :

- calculer le D84 moyen sur la section aval pour le débit observé,

- déterminer l'évolution des hauteurs d'eau moyennes en fonction du débit, connaissant la perte de charge linéaire (égale à la pente selon la loi de profondeur normale) et le D84 calculé précédemment.

\section{Sur les autres sections}

La formule de Limerinos est ensuite utilisée dans un bilan de quantité de mouvement (basé sur les équations de Saint-Venant) entre deux sections consécutives, en remontant section par section de l'aval vers l'amont. Le modèle calcule successivement :

- les D84 moyens de chaque section au débit observé,

- la cote de l'eau, la largeur mouillée et les vitesses moyennes sur chaque section et pour différents débits simulés.

\section{Redistribution latérale des vitesses}

A l'issue de la reconstitution de la ligne d'eau, il importe pour quantifier la capacité d'accueil biologique d'une station, d'estimer les valeurs ponctuelles des vitesses dans chaque cellule.

La formule de Limerinos est à nouveau utilisée pour :

- calculer, au débit observé, les D84 ponctuels de chaque cellule à partir des vitesses ponctuelles observées,

- estimer, pour les débits simulés, les vitesses ponctuelles à partir des D84 ponctuels (supposés constants) et de la perte de charge de la section. Lorsqu'il s'agit d'un point situé hors d'eau au débit observé, le D84 ponctuel est estimé par la moyenne des D84 des deux points immergés les plus proches.

A partir de l'ensemble des vitesses reconstituées pour un transect, une valeur de débit est calculée et comparée avec le débit simulé. Le rapport entre ces deux valeurs est utilisé comme coefficient correcteur de chaque vitesse ponctuelle.

Cette procédure a été validée à partir de mesures réalisées à plusieurs débits (MALAVOI, 1991). Plus le débit simulé est élevé, moins la redistribution des vitesses est fiable localement, surtout dans le cas des faciès très chaotiques; elle reste cependant acceptable sur l'ensemble de la section (MALAVOI, 1991). 